\title{
A PARAMETRIZED DE RHAM DECOMPOSITION THEOREM FOR CR MANIFOLDS
}

\author{
ATSUSHI HAYASHIMOTO
}

(Received June 11, 2012, revised May 9, 2013)

\begin{abstract}
The CR equivalence problem between CR manifolds with slice structure is studied. Let $N$ be a connected holomorphically nondegenerate real analytic hypersurface and $M(p)$ a finitely nondegenerate real analytic hypersurface parametrized by $p \in N$. Let $M$ be a totality of $N$ and $M(p)$ with moving $p$ in $N$. Assume that $M$ and $\widetilde{M}$ (with a same structure as $M)$ are CR equivalent and that $N$ and $\tilde{N}$ are also CR equivalent. Then we prove that, for any $p \in N$, there exists $\tilde{p} \in \tilde{N}$ such that $M(p)$ is CR equivalent to $\tilde{M}(\tilde{p})$.
\end{abstract}

1. Introduction. In 1952, de Rham proved the decomposition theorem of Riemannian manifolds, which states that a simply connected and complete Riemannian manifold $M$ is isometric to the direct product $M_{0} \times M_{1} \times \cdots \times M_{k}$, where $M_{0}$ is a Euclidean space (possibly of dimension 0 ) and $M_{1}, \ldots, M_{k}$ are all simply connected, complete, irreducible Riemannian manifolds. Such a decomposition is unique up to ordering.

The purpose of this article is to study a parameter version of this theorem in CR geometry.

We denote $z^{\prime}=\left(z_{1}, \ldots, z_{m}\right) \in \boldsymbol{C}^{m}, z=\left(z^{\prime}, z_{m+1}\right)$ and $w^{\prime}=\left(w_{1}, \ldots, w_{n}\right) \in \boldsymbol{C}^{n}, w=$ $\left(w^{\prime}, w_{n+1}\right)$. Now we shall define a CR manifold with slices $M(p)$ over a base space $N$. Let $N \subset C^{n+1}$ be a real analytic hypersurface. Then, by the implicit function theorem, we may assume that it is defined by the equation

$$
w_{n+1}=Q^{2}\left(w^{\prime}, \bar{w}^{\prime}, \bar{w}_{n+1}\right)
$$

in the normal coordinate for a real analytic function $Q^{2}$. Take $w \in \boldsymbol{C}^{n+1}$ and let $M(w) \subset$ $C^{m+1}$ be a real analytic hypersurface with real analytic parameters $w$ and $\bar{w}$. Then by the coordinate change which is biholomorphic in $z$ and is real analytic in $w$ and $\bar{w}$, we may assume that its defining function is of the form

$$
z_{m+1}=Q^{1}\left(z^{\prime}, \bar{z}^{\prime}, \bar{z}_{m+1} ; w, \bar{w}\right)
$$

in the normal coordinate in $z$ and $\bar{z}$, where $Q^{1}$ is a real analytic function.

Definition 1.1. Let $M \subset \boldsymbol{C}^{m+n+2}$ be a CR manifold. We say that $M$ is a CR manifold with slices $M(p) \subset \boldsymbol{C}^{m+1}$ over a base space $N \subset \boldsymbol{C}^{n+1}$ for $p \in N$ if it is constructed by

$$
M=\bigcup_{p \in N}(M(p) \times\{p\}) .
$$

2000 Mathematics Subject Classification. Primary 32V20; Secondary 32V35.

Key words and phrases. CR equivalence, Segre mapping, finite nondegeneracy, holomorphic nondegeneracy. 
Here $N$ is defined by the equation (1) and $M(p)$ is by (2) with $w=p \in N$.

We will consider the case where there exists a point $q \in \boldsymbol{C}^{m+1}$ such that, for any $p \in$ $N$, the point $q$ is contained in $M(p)$. In this case, we have $M=\bigcup_{p \in N}(M(p) \times\{p\}) \supset$ $\bigcup_{p \in N}(\{q\} \times\{p\})=\{q\} \times N$.

In this paper, we assume that all objects are in $C^{\omega}$ smooth. Let $A$ be a certain object defined on the source space. Then we denote by $\widetilde{A}$ a corresponding object on the target space with the same properties as $A$.

The main theorem of this article is the following. The definition of terms in the statement are given in Section 2.

THEOREM 1.2. Let $M \subset C^{m+n+2}$ be a CR manifold with slices $M(p)$ over a base space $N$, where $N \subset C^{n+1}$ is a holomorphically nondegenerate connected real hypersurface defined by (1) and $M(p) \subset C^{m+1}$ is a real hypersurface defined by (2) with $C^{\omega}$ parametrization by $p \in N$. Assume that there exists $q \in C^{m+1}$ such that, for any $p \in N, q$ is contained in $M(p)$ and $M(p)$ is finitely nondegenerate at $q$. Assume $M$ (resp. $N$ ) is CR equivalent to $\widetilde{M}$ (resp. $\widetilde{N})$ by a CR diffeomorphism $H$ (resp. $\left.H\right|_{\{q\} \times N}$ ). Then, for any $p \in N$, there exists $\tilde{p} \in \tilde{N}$ such that $M(p)$ is CR equivalent to $\tilde{M}(\tilde{p})$.

There are two comments.

1. This theorem claims that if the total manifold consists of a parameter space and slices on it and if such two total manifolds are CR equivalent and parameter spaces are also $\mathrm{CR}$ equivalent, then we can conclude that a slice of a manifold is mapped $\mathrm{CR}$ equivalently into a slice of the other.

2. Since we work under the hypothesis of a holomorphically nondegeneracy and use the theorem of identity in Lemmas 3.4 and 3.6, the regularity of real analyticity can not be weakened to be infinitely differentiable.

If all slices do not depend on $p \in N$, the total manifold $M$ is just the direct product of the base space and a slice. We have already proved the case in [9].

THEOREM 1.3. Let $(M, p)$ be a germ of a smooth CR-manifold which is finitely nondegenerate and of finite type on a dense subset. Then, up to permutations, there exists a unique decomposition

$$
(M, p) \cong\left(M_{1}, p_{1}\right) \times \cdots \times\left(M_{m}, p_{m}\right)
$$

where each germ $\left(M_{j}, p_{j}\right)$ is irreducible. Furthermore, if $f$ is (the germ of ) a smooth local CR-diffeomorphism between $(M, p)$ and (the germ of) another smooth CR-manifold $\left(M^{\prime}, p^{\prime}\right)$ and if $\left(M^{\prime}, p^{\prime}\right) \cong\left(M_{1}^{\prime}, p_{1}^{\prime}\right) \times \cdots \times\left(M_{m^{\prime}}^{\prime}, p_{m^{\prime}}\right)$ is the corresponding decomposition into irreducible factors, then $m=m^{\prime}$ and, after a permutation of the factors $\left(M_{j}^{\prime}, p_{j}^{\prime}\right), f$ is factorized as a direct product of the form $f=f^{1} \times \cdots \times f^{m}$, where $f^{j}:\left(M_{j}, p_{j}\right) \rightarrow$ $\left(M_{j}^{\prime}, p_{j}^{\prime}\right)$ are (germs of ) local CR-diffeomorphisms for $j=1, \ldots, m$. 
A parameter version of this decomposition theorem was also obtained by the author. The worm hypersurface $W_{h}$ is defined by

$$
W_{h}=\left\{(z, w) \in C^{2}:|z-\exp (i h(w, \bar{w}))|^{2}-1=0, h \text { is a harmonic function }\right\} .
$$

This is parametrized by a harmonic function $h(w, \bar{w})$ and is of infinite type along $\{z=0\}$ in the sense of Bloom and Graham [4]. When we fix $w=w_{0}$, a part of a circle centered at $\exp \left(i h\left(w_{0}, \bar{w}_{0}\right)\right)$ and radius one, which we denote by $W_{h}\left(w_{0}\right)$, appears. $W_{h}\left(w_{0}\right)$ goes around along complex variety $\{z=0\}$ as $w_{0}$ moves. Therefore the hypersurface $W_{h}$ consists of a base space $\{z=0\}$ and slices $W_{h}(w)$. About a CR equivalence problem for such hypersurfaces, the author proved the following theorem in [8].

THEOREM 1.4. Let $(f, g): W_{h} \rightarrow W_{h^{\prime}}$ be a real analytic CR equivalence mapping near the origin between worm hypersurfaces parametrized by harmonic functions $h$ and $h^{\prime}$. Then $f$ and $g$ are expanded in a standard coordinate in $C^{2}$ as

$$
f(z, w)=z, g(z, w)=\sum_{q \geq 1} b_{q} w^{q} .
$$

Since $\{z=0\}$ is mapped to $\{z=0\}$ and $W_{h}(w)$ to $W_{h^{\prime}}(g(w))$, this theorem is regarded as a parameter version of [9].

This condition in Theorem 1.2 is called "slice preserving" or "leaf preserving" and they appear in other subjects. For example, the Rudin theorem and the Baracco-Zampieri theorem in complex analysis, which we mention in Section 4, are given with such condition. This situation also appears in differential geometry and the theory of differential equation. Let $\mathcal{F}$ be a $C^{\infty}$-foliation on a compact $C^{\infty}$-manifold. Fukui [7] studied the group of all leaf preserving $C^{\infty}$-diffeomorphisms of $(M, \mathcal{F})$ which are isotopic to the identity through leaf preserving $C^{\infty}$-diffeomorphisms. He obtained the necessary and sufficient condition for such a group to be simple. As a problem of differential equation, the group-invariant solutions have been studied. We say that the group $G$ acts projectably on the open set where independent and dependent variables belong if the action of $G$ is of the form $(\tilde{u}, \tilde{x})=(\varphi(x, u), \phi(x))$. In view of Idea 3.1, this is the form that we want to prove. Construct a system of partial differential equations whose solutions are the complexified defining functions of $M$ in Theorem 1.2. Then a CR automorphism group of the manifold under consideration is a totally real part of the symmetry group of the system. Therefore by comparing this case with ours, Theorem 1.2 shows that the action of the symmetry group of such a system is projectable.

Here is an outline of this paper. In Section 2, we give some definition in the general situation and specify them for our setting in Subsection 3.1. In Subsection 3.2, we prove the main theorem along the line on Idea 3.1. Finally in Section 4, we pose two questions related to "slice preserving" and "level set preserving" situations.

A part of this work was done while the author stayed in the University of California, San Diego. He expresses his deep thanks to Professors P. Ebenfelt and M. S. Baouendi for useful discussion. 
2. Nondegeneracy conditions and Segre mappings. Let $M$ be a real analytic CR submanifold of codimension $d$ in $\boldsymbol{C}^{N}$. We take a coordinate $Z=(z, w)$ in $\boldsymbol{C}^{N}$ near the origin with $z=\left(z_{1}, \ldots, z_{n}\right), w=\left(w_{1}, \ldots, w_{d}\right), n+d=N$. Let $\rho=\left(\rho_{1}, \ldots, \rho_{d}\right)$ be a defining function for $M$. By implicit function theorem, we may assume that $M$ is defined by the equations $w_{j}=Q_{j}(z, \bar{z}, \bar{w}), j=1, \ldots, d$.

2.1. Nondegeneracy conditions. In this subsection, we give definitions of nondegeneracy conditions in the general setting. See [1] for more detail.

By a holomorphic vector field, we mean a $(1,0)$-type vector field $X$ of the form

$$
X=\sum_{j=1}^{N} a_{j}(Z) \frac{\partial}{\partial Z_{j}}
$$

with holomorphic functions $a_{j}$.

DEFINITION 2.1. A real analytic CR manifold $M$ is holomorphically nondegenerate at $p$ if there is no holomorphic vector field $X$ tangent to $M$ near $p$ such that $\left.X\right|_{M} \not \equiv 0$.

Note that if $M$ is connected and is holomorphically nondegenerate at a point $p$, then it is holomorphically nondegenerate at every point. So we shall omit the reference point.

Let $\left\{L_{1}, \ldots, L_{n}\right\}$ be a local basis for the CR vector fields of type $(0,1)$. For a multi-index $\alpha=\left(\alpha_{1}, \ldots, \alpha_{n}\right)$, we use the notation

$$
L^{\alpha}=L_{1}^{\alpha_{1}} \cdots L_{n}^{\alpha_{n}}
$$

We also use the notation

$$
\frac{\partial \rho_{j}}{\partial Z}=\left(\frac{\partial \rho_{j}}{\partial Z_{1}}, \ldots, \frac{\partial \rho_{j}}{\partial Z_{N}}\right) .
$$

DEFINITION 2.2. $\quad M$ is $k$-nondegenerate at $p$ if

$$
\operatorname{span}\left\{L^{\alpha}\left(\frac{\partial \rho_{j}}{\partial Z}\right)(p, \bar{p}): j \in\{1, \ldots, d\},|\alpha| \leq k\right\}=C^{N} .
$$

If there exists such a finite integer $k, M$ is said to be finitely nondegenerate at $p$.

In the hypersurface case, we have the following relations.

FACT 2.3. Let $M$ be a real analytic connected hypersurface. Consider the following three conditions.

(a) $M$ is holomorphically nondegenerate.

(b) There exist $p \in M$ and $k>0$ such that $M$ is $k$-nondegenerate at $p$.

(c) $M$ is of finite type at $p$ (for definition, see [4]).

Then (a) is equivalent of (b), and (b) implies (c).

2.2. Segre mapping. In this subsection, we define a Segre mapping. The reader is referred to [2], [3] and [6] for the definition and properties of Segre mappings.

Let $M, \widetilde{M} \subset C^{N}$ be real analytic CR submanifolds, and $H: M \rightarrow \widetilde{M}$ a real analytic CR mapping. We write $H=(F, G)$ for the coordinate $(\tilde{z}, \tilde{w}) \in \boldsymbol{C}^{n} \times \boldsymbol{C}^{d}$. Since the mapping 
$(F, G)$ sends $M$ into $\tilde{M}$, they satisfy

$$
G(z, w)=\widetilde{Q}(F(z, w), \bar{F}(\chi, \tau), \bar{G}(\chi, \tau))
$$

and its complex conjugate

$$
\bar{G}(\chi, \tau)=\bar{Q}(\bar{F}(\chi, \tau), F(z, w), G(z, w)),
$$

for $(z, w, \chi, \tau) \in C^{N} \times C^{N}$ with

$$
w=Q(z, \chi, \tau) .
$$

For a positive integer $k$, we define a mapping $u^{k}: \boldsymbol{C}^{k n} \rightarrow \boldsymbol{C}^{d}$ inductively by

$$
u^{1}\left(t^{1}\right)=0, u^{k}\left(t^{1}, \ldots, t^{k}\right)=Q\left(t^{k}, t^{k-1}, \overline{u^{k-1}}\left(t^{1}, \ldots, t^{k-1}\right)\right), k \geq 2,
$$

for $t^{j}=\left(t_{1}^{j}, \ldots, t_{n}^{j}\right) \in \boldsymbol{C}^{n}$. For simplicity, we use the notation $t^{[k]}=\left(t^{1}, \ldots, t^{k}\right) \in \boldsymbol{C}^{k n}$.

Definition 2.4. The $k$ th Segre mapping of $M$ at 0 is the mapping $v^{k}: C^{k n} \rightarrow C^{N}$ defined by

$$
\boldsymbol{C}^{k n} \ni t^{[k]} \mapsto v^{k}\left(t^{[k]}\right)=\left(t^{k}, u^{k}\left(t^{[k]}\right)\right) \in \boldsymbol{C}^{N} .
$$

This mapping has a close relation to the finite type condition.

FACT 2.5. Let $M$ be of finite type in the sense of Bloom-Graham and $v^{k}$ its Segre mapping. Then, for a sufficiently large $k, v^{k}$ has a full rank. Therefore, together with Fact 2.3, the image under the Segre mapping $v^{k}$ of the finitely nondegenerate hypersurface contains an open subset of $\boldsymbol{C}^{N}$.

For example, if $k \geq 3$ is odd, we have

$$
\begin{aligned}
u^{k}\left(t^{[k]}\right) & =Q\left(t^{k}, t^{k-1}, \bar{Q}\left(t^{k-1}, t^{k-2}, Q\left(t^{k-2}, t^{k-3}, \ldots, \bar{Q}\left(t^{2}, t^{1}, 0\right), \ldots\right)\right)\right) \\
& =Q\left(t^{k}, \overline{v^{k-1}}\left(t^{[k-1]}\right)\right) .
\end{aligned}
$$

By the definition of Segre mapping, (11) and (12) hold for

$$
(z, w ; \chi, \tau)=\left(v^{k}\left(t^{[k]}\right) ; \overline{v^{k-1}}\left(t^{[k-1]}\right)\right)
$$

and

$$
(z, w ; \chi, \tau)=\left(v^{k-2}\left(t^{[k-2]}\right) ; \overline{v^{k-1}}\left(t^{[k-1]}\right)\right)
$$

for any $k \geq 2$. Substituting (17) into (11), and (18) into (12), we have identities

$$
\begin{aligned}
& G \circ v^{k}=\widetilde{Q}\left(F \circ v^{k}, \overline{F \circ v^{k-1}}, \overline{G \circ v^{k-1}}\right), \\
& \overline{G \circ v^{k-1}}=\widetilde{\widetilde{Q}}\left(\overline{F \circ v^{k-1}}, F \circ v^{k-2}, G \circ v^{k-2}\right) .
\end{aligned}
$$

Then substitute (20) into (19). Continuing this process for $l=k-2, k-3, \ldots, 1$, we obtain, for any $k \geq 1$ odd,

$$
\begin{aligned}
G \circ v^{k}= & \widetilde{Q}\left(F \circ v^{k}, \overline{F \circ v^{k-1}}, \widetilde{Q}\left(\overline{F \circ v^{k-1}}, F \circ v^{k-2},\right.\right. \\
& \left.\left.\widetilde{Q}\left(\ldots, \overline{\widetilde{Q}}\left(\overline{F \circ v^{2}}, F \circ v^{1}, G \circ v^{1}\right), \ldots\right)\right)\right),
\end{aligned}
$$


and for $k \geq 2$ even,

$$
\begin{aligned}
G \circ v^{k}= & \widetilde{Q}\left(F \circ v^{k}, \overline{F \circ v^{k-1}}, \widetilde{\widetilde{Q}}\left(\overline{F \circ v^{k-1}}, F \circ v^{k-2},\right.\right. \\
& \left.\widetilde{Q}\left(\ldots, \widetilde{Q}\left(F \circ v^{2}, \overline{F \circ v^{1}}, \overline{G \circ v^{1}}\right), \ldots\right)\right),
\end{aligned}
$$

where $F \circ v^{j}=\left(F \circ v^{j}\right)\left(t^{[j]}\right)$ and $\overline{F \circ v^{j}}=\left(\overline{F \circ v^{j}}\right)\left(t^{[j]}\right)$. We shall omit the variables of $v^{k}$ if there is no confusion.

3. Proof of Theorem 1.2. Let $H$ be a CR diffeomorphism between $M$ and $\widetilde{M}$ as in Theorem 1.2, and decompose it as $H(z, w)=\left(F_{1}, \ldots, F_{m+1}, G_{1}, \ldots, G_{n+1}\right)(z, w)$ corresponding to the coordinate for $\tilde{M}$. The following is the main idea to prove the theorem.

IDEA 3.1. If we can prove that $G_{1}, \ldots, G_{n+1}$ are independent of the variables $z_{1}, \ldots, z_{m+1}$, then Theorem 1.2 is true.

Substitute the defining function (1) of $N$ into (2) of $M(w)$ for $w \in N$. Then we can eliminate $w_{n+1}$ from (2) and we may assume that the defining function of $M(w)$ is of the form:

$$
z_{m+1}=Q^{1}\left(z^{\prime}, \bar{z}^{\prime}, \bar{z}_{m+1} ; w^{\prime}, \bar{w}^{\prime}, \bar{w}_{n+1}\right) .
$$

3.1. Segre mappings in our setting. First, we fix the notation for our purpose. We correspond the variable $t^{k}$ (resp. $\left.s^{k}\right)$ of the Segre mapping to $z^{\prime}=\left(z_{1}, \ldots, z_{m}\right)$ (resp. $w^{\prime}=$ $\left(w_{1}, \ldots, w_{n}\right)$ ). Since $N$ is holomorphically nondegenerate, there exists a point where $N$ is finitely nondegenerate (cf. Fact 2.3). We take the origin as the reference point.

We shall denote by $v_{N}^{k}, v_{M(p)}^{k}$ and $V^{k}$ the $k$ th Segre mappings of $N, M(p)$ and $M$, respectively which are defined inductively as follows:

$$
\begin{gathered}
v_{N}^{1}\left(s^{1}\right)=\left(s^{1}, 0\right), \\
v_{N}^{k}\left(s^{[k]}\right)=\left(s^{k}, Q^{2}\left(s^{k}, \overline{v_{N}^{k-1}}\left(s^{[k-1]}\right)\right)\right), \\
v_{M(p)}^{1}\left(t^{1}, s^{1}\right)=\left(t^{1}, 0\right), \\
v_{M(p)}^{k}\left(t^{[k]}, s^{[k]}\right)=\left(t^{k}, Q^{1}\left(t^{k}, \overline{v_{M(p)}^{k-1}}\left(t^{[k-1]}, s^{[k-1]}\right) ; s^{k}, \overline{v_{N}^{k-1}}\left(s^{[k-1]}\right)\right)\right), \\
V^{k}\left(t^{[k]}, s^{[k]}\right)=\left(v_{M(p)}^{k}\left(t^{[k]}, s^{[k]}\right), v_{N}^{k}\left(s^{[k]}\right)\right) .
\end{gathered}
$$

Now we need a remark on this notation. Since $v_{M(p)}^{k}$ depends on $p \in N$, we should write $v_{M(p)}^{k}\left(t^{[k]}, s_{p}^{[k]}\right)$ instead of $v_{M(p)}^{k}\left(t^{[k]}, s^{[k]}\right)$. But since the point $p \in N$ is arbitrary, we may omit $p$ from $s_{p}^{[k]}$. This may not give any confusion. Since $M$ is finitely nondegenerate, the image under $V^{k}$ contains an open subset of $\boldsymbol{C}^{n+m+2}$.

First we write down the equation (21) or (22) for $\left(G_{1}, \ldots, G_{n+1}\right): M \rightarrow \widetilde{N}$ as

$$
\begin{aligned}
G_{n+1} \circ V^{k}= & \widetilde{Q^{2}}\left(G^{\prime} \circ V^{k}, \overline{G^{\prime} \circ V^{k-1}}, \widetilde{Q^{2}}\left(\overline{G^{\prime} \circ V^{k-1}}, G^{\prime} \circ V^{k-2},\right.\right. \\
& \left.\left.\widetilde{Q^{2}}\left(G^{\prime} \circ V^{k-2}, \overline{G^{\prime} \circ V^{k-3}}, \overline{\widetilde{Q}^{2}}(\ldots)\right)\right)\right) .
\end{aligned}
$$


for $G^{\prime}=\left(G_{1}, \ldots, G_{n}\right)$. We restrict this equation to $t^{k-3}=t^{k-1}$ and $s^{k-3}=s^{k-1}$. Then the $k$ th and the $(k-1)$ st restricted Segre mappings, on which we add $\widehat{a}$, are written as

$$
\begin{gathered}
\overline{\hat{v}_{M(p)}^{k-1}}=\left(t^{k-1}, \overline{Q^{1}}\left(t^{k-1}, v_{M(p)}^{k-4} ; s^{k-1}, v_{N}^{k-4}\right)\right), \\
\overline{\hat{v}_{N}^{k-1}}=\left(s^{k-1}, \overline{Q^{2}}\left(s^{k-1}, v_{N}^{k-4}\right)\right), \\
\hat{v}_{M(p)}^{k}=\left(t^{k}, Q^{1}\left(t^{k}, t^{k-1}, \overline{Q^{1}}\left(t^{k-1}, v_{M(p)}^{k-4} ; s^{k-1}, v_{N}^{k-4}\right) ; s^{k}, \overline{\hat{v}_{N}^{k-1}}\right)\right), \\
\hat{v}_{N}^{k}=\left(s^{k}, Q^{2}\left(s^{k}, s^{k-1}, \overline{Q^{2}}\left(s^{k-1}, v_{N}^{k-4}\right)\right)\right) .
\end{gathered}
$$

Therefore, the equation (29) restricted to $t^{k-3}=t^{k-1}$ and $s^{k-3}=s^{k-1}$ is written as

$$
\begin{aligned}
G_{n+1} \circ \widehat{V}^{k}= & \widetilde{Q^{2}}\left(G^{\prime} \circ \widehat{V}^{k}, \overline{G^{\prime} \circ \widehat{V}^{k-1}}, \overline{\widetilde{Q}^{2}}\left(\overline{G^{\prime} \circ \widehat{V}^{k-1}}, G^{\prime} \circ V^{k-4},\right.\right. \\
& \left.\left.\widetilde{Q^{2}}\left(G^{\prime} \circ V^{k-4}, \overline{G^{\prime} \circ V^{k-5}}, \widetilde{\widetilde{Q}^{2}}(\ldots)\right)\right)\right) .
\end{aligned}
$$

We introduce the variables $T$ and $S$ as

$$
\begin{aligned}
T= & \left(G^{\prime} \circ \widehat{V}^{k}, \overline{G^{\prime} \circ \widehat{V}^{k-1}}, \widetilde{Q^{2}}\left(\overline{G^{\prime} \circ \widehat{V}^{k-1}}, G^{\prime} \circ V^{k-4},\right.\right. \\
& \left.\left.\widetilde{Q^{2}}\left(G^{\prime} \circ V^{k-4}, \overline{G^{\prime} \circ V^{k-5}}, \widetilde{\widetilde{Q}^{2}}(\ldots)\right)\right)\right),
\end{aligned}
$$

$$
S=\left(\overline{G^{\prime} \circ \widehat{V}^{k-1}}, G^{\prime} \circ V^{k-4}, \widetilde{Q^{2}}\left(G^{\prime} \circ V^{k-4}, \overline{G^{\prime} \circ V^{k-5}}, \widetilde{Q^{2}}(\ldots)\right)\right)
$$

Take any $j=1, \ldots, m$ and fix it. Some indices which appear later may depend on $j$, but we shall not refer it. Differentiate (34) with respect to $t_{j}^{k}$ and $t_{j}^{k-1}$. Then we obtain the identities

$$
\begin{aligned}
\frac{\partial G_{n+1}}{\partial z_{j}} \circ & \widehat{V}^{k}+\frac{\partial G_{n+1}}{\partial z_{m+1}} \circ \widehat{V}^{k} \\
& \times \frac{\partial Q^{1}\left(t^{k}, t^{k-1}, \overline{Q^{1}}\left(t^{k-1}, v_{M(p)}^{k-4} ; s^{k-1}, v_{N}^{k-4}\right) ; s^{k}, \overline{\hat{v}_{N}^{k-1}}\right)}{\partial t_{j}^{k}} \\
= & \sum_{l=1}^{n} \frac{\partial \widetilde{Q^{2}}}{\partial w_{l}}(T)\left\{\frac{\partial G_{l}}{\partial z_{j}} \circ \widehat{V}^{k}+\frac{\partial G_{l}}{\partial z_{m+1}} \circ \widehat{V}^{k}\right. \\
& \left.\times \frac{\partial Q^{1}\left(t^{k}, t^{k-1}, \overline{Q^{1}}\left(t^{k-1}, v_{M(p)}^{k-4} ; s^{k-1}, v_{N}^{k-4}\right) ; s^{k}, \overline{\hat{v}_{N}^{k-1}}\right)}{\partial t_{j}^{k}}\right\},
\end{aligned}
$$




$$
\begin{aligned}
\frac{\partial G_{n+1}}{\partial z_{m+1}} \circ & \widehat{V}^{k} \frac{\partial Q^{1}\left(t^{k}, t^{k-1}, \overline{Q^{1}}\left(t^{k-1}, v_{M(p)}^{k-4} ; s^{k-1}, v_{N}^{k-4}\right) ; s^{k}, \overline{\hat{v}_{N}^{k-1}}\right)}{\partial t_{j}^{k-1}} \\
= & \sum_{l=1}^{n} \frac{\partial \widetilde{Q}^{2}}{\partial w_{l}}(T) \frac{\partial G_{l}}{\partial z_{m+1}} \circ \widehat{V}^{k} \\
& \times \frac{\partial Q^{1}\left(t^{k}, t^{k-1}, \overline{Q^{1}}\left(t^{k-1}, v_{M(p)}^{k-4} ; s^{k-1}, v_{N}^{k-4}\right) ; s^{k}, \overline{\hat{v}_{N}^{k-1}}\right)}{\partial t_{j}^{k-1}} \\
& +\sum_{l=1}^{n} \frac{\partial \overline{G_{l} \circ \widehat{V}^{k-1}}}{\partial t_{j}^{k-1}}\left\{\frac{\partial \widetilde{Q}^{2}}{\partial \bar{w}_{l}}(T)+\frac{\partial \widetilde{Q}^{2}}{\partial \bar{w}_{n+1}}(T) \frac{\partial \widetilde{Q}^{2}}{\partial \bar{w}_{l}}(S)\right\} .
\end{aligned}
$$

3.2. Independency of $z$. In this subsection, we shall prove that $G_{1} \circ \widehat{V}^{k}\left(t^{[k]}\right.$, $\left.s^{[k]}\right), \ldots, G_{n+1} \circ \widehat{V}^{k}\left(t^{[k]}, s^{[k]}\right)$ are independent of $t^{k}$, which will lead to the independency of $z$. Restrict the identity (38) to the variety

$$
W_{j}=\left\{\left(t^{k}, t^{k-1}, t^{[k-4]}, s^{k}, s^{k-1}, s^{[k-4]}\right):\right.
$$

$$
\left.\frac{\partial Q^{1}\left(t^{k}, t^{k-1}, \overline{Q^{1}}\left(t^{k-1}, v_{M(p)}^{k-4} ; s^{k-1}, v_{N}^{k-4}\right) ; s^{k}, \overline{\hat{v}_{N}^{k-1}}\right)}{\partial t_{j}^{k-1}}=0\right\} .
$$

Then it becomes

$$
\sum_{l=1}^{n} \frac{\partial \overline{G_{l} \circ \widehat{V}^{k-1}}}{\partial t_{j}^{k-1}}\left\{\frac{\partial \widetilde{Q^{2}}}{\partial \bar{w}_{l}}(T)+\frac{\partial \widetilde{Q^{2}}}{\partial \bar{w}_{n+1}}(T) \frac{\partial \widetilde{\widetilde{Q}^{2}}}{\partial \bar{w}_{l}}(S)\right\}=0 .
$$

There exists $j_{0}$ depending on $j$ such that

$$
\frac{\partial^{2} Q^{1}\left(t^{k}, t^{k-1}, \overline{Q^{1}}\left(t^{k-1}, v_{M(p)}^{k-4} ; s^{k-1}, v_{N}^{k-4}\right) ; s^{k}, \overline{\hat{v}_{N}^{k-1}}\right)}{\partial t_{j_{0}}^{k} \partial t_{j}^{k-1}} \neq \equiv
$$

near the points where all variables are zero except for $t^{k-1}$. We may assume that $j_{0}=1$. We define vector fields $X^{i}, i=1, \ldots, n$, on $W_{j}$ as

$$
X^{i}=\frac{\partial^{2} Q^{1}}{\partial t_{1}^{k} \partial t_{j}^{k-1}} \frac{\partial}{\partial s_{i}^{k}}-\frac{\partial^{2} Q^{1}}{\partial s_{i}^{k} \partial t_{j}^{k-1}} \frac{\partial}{\partial t_{1}^{k}} .
$$

We introduce the following notation.

$$
\begin{gathered}
A_{\alpha_{1}, \ldots, \alpha_{\lambda}}^{i_{1}, \ldots, i_{\lambda}}=X^{i_{1}} G_{\alpha_{1}} \circ \widehat{V}^{k} \times \cdots \times X^{i_{\lambda}} G_{\alpha_{\lambda}} \circ \widehat{V}^{k}, \\
q_{l}=\frac{\partial \widetilde{Q^{2}}}{\partial \bar{w}_{l}}(T)+\frac{\partial \widetilde{Q^{2}}}{\partial \bar{w}_{n+1}}(T) \frac{\partial \widetilde{Q^{2}}}{\partial \bar{w}_{l}}(S),
\end{gathered}
$$




$$
q_{l ; \alpha_{1}, \ldots, \alpha_{\lambda}}=\frac{\partial^{\lambda+1} \widetilde{Q}^{2}}{\partial \bar{w}_{l} \partial w_{\alpha_{1}} \cdots \partial w_{\alpha_{\lambda}}}(T)+\frac{\partial^{\lambda+1}{\widetilde{Q^{2}}}^{2}}{\partial \bar{w}_{n+1} \partial w_{\alpha_{1}} \cdots \partial w_{\alpha_{\lambda}}}(T) \frac{\partial \widetilde{Q^{2}}}{\partial \bar{w}_{l}}(S) .
$$

Let $A(\lambda)$ be an $n^{\lambda} \times n^{\lambda}$ matrix with element $A_{\alpha_{1}, \ldots, \alpha_{\lambda}}^{i_{1}, \ldots, i_{\lambda}}$ as the $\left(\left(i_{1}-1\right) n^{\lambda-1}+\left(i_{2}-1\right) n^{\lambda-2}\right.$ $\left.+\cdots+\left(i_{\lambda-1}-1\right) n+i_{\lambda},\left(\alpha_{1}-1\right) n^{\lambda-1}+\left(\alpha_{2}-1\right) n^{\lambda-2}+\cdots+\left(\alpha_{\lambda-1}-1\right) n+\alpha_{\lambda}\right)$-th component for $i_{1}, \ldots, i_{\lambda}, \alpha_{1}, \ldots, \alpha_{\lambda}=1, \ldots, n$. If we apply $X^{1}, \ldots, X^{n}$ to the vector $\left(q_{1}, \ldots, q_{n}\right) K$ times, then we have $n^{K}$ vectors. We order them in the following way. The $\left(\left(a_{1}-1\right) n^{K-1}+\right.$ $\left.\left(a_{2}-1\right) n^{K-2}+\cdots+\left(a_{K-1}-1\right) n+a_{K}\right)$-th vector is $\left(X^{a_{1}} \cdots X^{a_{K}} q_{1}, \ldots, X^{a_{1}} \cdots X^{a_{K}} q_{n}\right)$. We order the $n^{\lambda}$ vectors $\left\{\left(q_{1 ; \alpha_{1}, \ldots, \alpha_{\lambda}}, \ldots, q_{n ; \alpha_{1}, \ldots, \alpha_{\lambda}}\right)\right\}_{\alpha_{1}, \ldots, \alpha_{\lambda}}$ for $\alpha_{1}, \ldots, \alpha_{\lambda}=1, \ldots, n$ in the same way. Then we obtain the matrix equation below by applying $X^{1}, \ldots, X^{n}$ to $\left(q_{1}, \ldots, q_{n}\right)$ $L$ times for sufficiently large $L$. The first matrix in the right-hand side is a lower triangle matrix whose diagonals are the matrices defined above.

$$
\begin{aligned}
& \left(\begin{array}{ccc}
X^{1} q_{1} & \cdots & X^{1} q_{n} \\
\vdots & \ddots & \vdots \\
X^{n} q_{1} & \cdots & X^{n} q_{n} \\
X^{1} X^{1} q_{1} & \cdots & X^{1} X^{1} q_{n} \\
\vdots & \ddots & \vdots \\
X^{n} X^{n} q_{1} & \cdots & X^{n} X^{n} q_{n} \\
\vdots & \ddots & \vdots \\
X^{1} \cdots X^{1} q_{1} & \cdots & X^{1} \cdots X^{1} q_{n} \\
\vdots & \ddots & \vdots \\
X^{n} \cdots X^{n} q_{1} & \cdots & X^{n} \cdots X^{n} q_{n}
\end{array}\right) \\
& =\left(\begin{array}{ccccc}
A(1) & & 0 & \\
& A(2) & & \\
* & & \ddots & \\
& & & A(L)
\end{array}\right)\left(\begin{array}{cccc}
q_{1 ; 1} & \ldots & q_{n ; 1} \\
\vdots & \ddots & \vdots \\
q_{1 ; n} & \ldots & q_{n ; n} \\
q_{1 ; 11} & \ldots & q_{n ; 11} \\
\vdots & \ddots & \vdots \\
q_{1 ; n n} & \ldots & q_{n ; n n} \\
\vdots & \ddots & \vdots \\
q_{1 ; 1 \ldots 1} & \ldots & q_{n ; 1 \ldots 1} \\
\vdots & \ddots & \vdots \\
q_{1 ; n, \ldots n} & \ldots & q_{n ; n, \ldots n}
\end{array}\right) .
\end{aligned}
$$

We denote by $(X q)$ the matrix in the left-hand side and by $(A)$ and by $(q)$ the first and the second matrices in the right-hand side. It follows from $\operatorname{det}(A)=\operatorname{det} A(1) \times \cdots \times \operatorname{det} A(L)$ and $\operatorname{det} A(\lambda+1)=(\operatorname{det} A(1))^{n^{\lambda}}(\operatorname{det} A(\lambda))^{n}$ that $\operatorname{det}(A) \not \equiv 0$ is equivalent to $\operatorname{det} A(1) \not \equiv 0$. 
LEMMA 3.2.

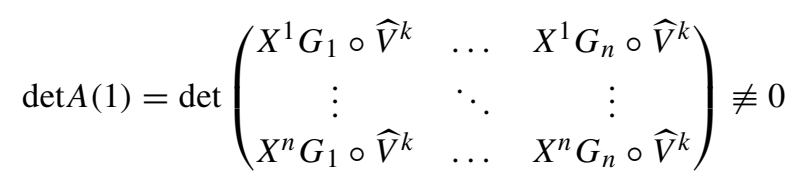

near the origin.

PROOF. Evaluate the matrix (47) at the points where all variables are zero except for $t^{k-1}$. Then it becomes

$$
\frac{\partial^{2} Q^{1}}{\partial t_{j}^{k-1} \partial t_{1}^{k}}\left(\begin{array}{ccc}
\frac{\partial G_{1}}{\partial w_{1}}(0) & \ldots & \frac{\partial G_{n}}{\partial w_{1}}(0) \\
\vdots & \ddots & \vdots \\
\frac{\partial G_{1}}{\partial w_{n}}(0) & \ldots & \frac{\partial G_{n}}{\partial w_{n}}(0)
\end{array}\right)
$$

Since $\left.G\right|_{N}$ is a CR diffeomorphism between $N$ and $\tilde{N}$ near the origin, and since we have (41), the determinant of (48) does not vanish identically.

We shall claim that $\operatorname{rank}(X q)=n$. Obviously, we have $\operatorname{rank}(X q) \leq n$. Next, we multiply the inverse matrix $(A)^{-1}$ to the both sides of (46), then we get

$$
n=\operatorname{rank}(q)=\operatorname{rank}(A)^{-1}(X q) \leq \operatorname{rank}(X q) .
$$

The first equality comes from the finite nondegeneracy of $N$ at the origin. Therefore, we conclude that $\operatorname{rank}(X q)=n$.

LEMMA 3.3. For any $k, \overline{G_{l} \circ \widehat{V}^{k-1}}$ is independent of $t_{j}^{k-1}$ on $W_{j}$ for $l=1, \ldots, n$.

Proof. By applying $X^{1}, \ldots, X^{n}$ to (40) step by step, we get

$$
\begin{aligned}
& \sum_{l=1}^{n} \frac{\partial \overline{G_{l} \circ \widehat{V}^{k-1}}}{\partial t_{j}^{k-1}} X^{i_{1}} q_{l}=0 \\
& \vdots \\
& \sum_{l=1}^{n} \frac{\partial \overline{G_{l} \circ \widehat{V}^{k-1}}}{\partial t_{j}^{k-1}} X^{i_{1}} \cdots X^{i_{\lambda}} q_{l}=0
\end{aligned}
$$

for sufficiently large $\lambda$. Write down these in a matrix equation as

$$
\left(\begin{array}{ccc}
X^{i_{1}} q_{1} & \ldots & X^{i_{1}} q_{n} \\
X^{i_{1}} X^{i_{2}} q_{1} & \ldots & X^{i_{1}} X^{i_{2}} q_{n} \\
\vdots & \ddots & \vdots \\
X^{i_{1}} \ldots X^{i_{\lambda}} q_{1} & \ldots & X^{i_{1}} \ldots X^{i_{\lambda}} q_{n}
\end{array}\right)\left(\begin{array}{c}
\frac{\partial \widehat{G_{1} \circ \widehat{V}^{k-1}}}{\partial t_{j}^{k-1}} \\
\vdots \\
\frac{\partial}{\partial G_{n} \circ \widehat{V}^{k-1}} \\
\partial t_{j}^{k-1}
\end{array}\right)=0 .
$$


The rank condition of $(X q)$ implies that any $\overline{G_{l} \circ \widehat{V}^{k-1}}$ is independent of $t_{j}^{k-1}$ on $W_{j}$ for any fixed $j$.

LEMMA 3.4. $G_{1}, \ldots, G_{n}$ are independent of $z_{1}, \ldots, z_{m+1}$.

Proof. By Lemma 3.3, we have

$$
\begin{aligned}
\frac{\partial \overline{G_{l} \circ \widehat{V}^{k-1}}}{\partial t_{j}^{k-1}} & =\frac{\partial \overline{G_{l}}}{\partial \bar{z}_{j}} \circ \overline{\widehat{V}^{k-1}}+\frac{\partial \overline{G_{l}}}{\partial \bar{z}_{n+1}} \circ \overline{\widehat{V}^{k-1}} \frac{\partial \overline{Q^{1}}\left(t^{k-1}, v_{M(p)}^{k-4} ; s^{k-1}, v_{N}^{k-4}\right)}{\partial t_{j}^{k-1}} \\
& =0
\end{aligned}
$$

on $W_{j}$. By restricting this equality to the variety

$$
Z_{j}=W_{j} \cap\left\{\frac{\partial \overline{Q^{1}}\left(t^{k-1}, v_{M(p)}^{k-4} ; s^{k-1}, v_{N}^{k-4}\right)}{\partial t_{j}^{k-1}}=0\right\},
$$

we get

$$
\frac{\partial \overline{G_{l}}}{\partial \bar{z}_{j}} \circ \overline{\widehat{V}^{k-1}}=0
$$

on $Z_{j}$. The following claim shows that $\widehat{V}^{k-1}\left(Z_{j}\right)$ contains an open set, which implies that $G_{l}$ does not depend on $z_{j}$.

CLAIM 3.5. The gradients by $t^{[k]}$ and $s^{[k]}$ of defining functions of $Z_{j}$ and each component of $\widehat{\widehat{V}}^{k-1}$ are linearly independent.

To show the claim, we prove that the gradients of $\partial \overline{Q^{1}}\left(t^{k-1}, v_{M(p)}^{k-4} ; s^{k-1}, v_{N}^{k-4}\right) / \partial t_{j}^{k-1}$ and $\overline{Q^{1}}\left(t^{k-1}, v_{M(p)}^{k-4} ; s^{k-1}, v_{N}^{k-4}\right)$ are linearly independent. Assume that they are not linearly independent, then there exists a function $R$ such that

$$
\begin{aligned}
& \left(\frac{\partial^{2} \overline{Q^{1}}\left(t^{k-1}, v_{M(p)}^{k-4} ; s^{k-1}, v_{N}^{k-4}\right)}{\partial t_{j}^{k-1} \partial t_{1}^{k-4}}, \ldots, \frac{\partial^{2} \overline{Q^{1}}\left(t^{k-1}, v_{M(p)}^{k-4} ; s^{k-1}, v_{N}^{k-4}\right)}{\partial t_{j}^{k-1} \partial t_{m}^{k-4}}\right) \\
& \quad=R\left(\frac{\partial \overline{Q^{1}}\left(t^{k-1}, v_{M(p)}^{k-4} ; s^{k-1}, v_{N}^{k-4}\right)}{\partial t_{1}^{k-4}}, \ldots, \frac{\partial \overline{Q^{1}}\left(t^{k-1}, v_{M(p)}^{k-4} ; s^{k-1}, v_{N}^{k-4}\right)}{\partial t_{m}^{k-4}}\right) .
\end{aligned}
$$

Since $M(p)$ is finitely nondegenerate, we can choose variables $t_{\alpha_{1}}^{k-4}, \ldots, t_{\alpha_{\lambda}}^{k-4}$ such that

$$
\frac{\partial^{\lambda+1} \overline{Q^{1}}\left(t^{k-1}, v_{M(p)}^{k-4} ; s^{k-1}, v_{N}^{k-4}\right)}{\partial t_{j}^{k-1} \partial t_{\alpha_{1}}^{k-4}, \ldots, \partial t_{\alpha_{\lambda}}^{k-4}}(0) \neq 0 .
$$

By differentiating the equality

$$
\frac{\partial^{2} \overline{Q^{1}}\left(t^{k-1}, v_{M(p)}^{k-4} ; s^{k-1}, v_{N}^{k-4}\right)}{\partial t_{j}^{k-1} \partial t_{\alpha_{1}}^{k-4}}=R \frac{\partial \overline{Q^{1}}\left(t^{k-1}, v_{M(p)}^{k-4} ; s^{k-1}, v_{N}^{k-4}\right)}{\partial t_{\alpha_{1}}^{k-4}}
$$


by $t_{\alpha_{2}}^{k-4}, \ldots, t_{\alpha_{\lambda}}^{k-4}$, we obtain

$$
\begin{aligned}
& \frac{\partial^{\lambda+1} \overline{Q^{1}}\left(t^{k-1}, v_{M(p)}^{k-4} ; s^{k-1}, v_{N}^{k-4}\right)}{\partial t_{j}^{k-1} \partial t_{\alpha_{1}}^{k-4}, \ldots, \partial t_{\alpha_{\lambda}}^{k-4}} \\
& =\text { (the summation of the differentials of } \overline{Q^{1}} \text { by } \\
& \left.\quad t_{\alpha_{1}}^{k-4}, \ldots, t_{\alpha_{\lambda}}^{k-4} \text { of order less than or equal to } \lambda\right),
\end{aligned}
$$

from which we reach a contradiction by evaluating at the origin. Therefore there does not exist such a function $R$, which shows the claim and $G_{l}$ is independent of $z_{j}$. Since $j$ is fixed arbitrary, we conclude that $G_{l}$ is independent of $z_{j}$ for $l=1, \ldots, n, j=1, \ldots, m$. Returning to the relation (53), we obtain that $G_{l}$ is independent of $z_{m+1}$ for $l=1, \ldots, n$.

LEMMA 3.6. $G_{n+1}$ is independent of $z_{1}, \ldots, z_{m+1}$.

Proof. By the identity (38) together with Lemma 3.4, we obtain that

$$
\frac{\partial G_{n+1}}{\partial z_{m+1}} \circ \widehat{V}^{k}=0 \text {. }
$$

Then by the identity (37), we get

$$
\frac{\partial G_{n+1}}{\partial z_{j}} \circ \widehat{V}^{k}=0
$$

These show that $G_{n+1}$ is independent of $z_{1}, \ldots, z_{m+1}$.

Now we have proved that $G_{1}, \ldots, G_{n+1}$ are independent of $z_{1}, \ldots, z_{m+1}$, which completes the proof of Theorem 1.2.

\section{Some questions related to other topics.}

4.1. The Rudin theorem. Rudin proved the following theorem in [10].

THEOREM 4.1. Suppose $n \geq 1, f: \boldsymbol{C}^{n} \rightarrow \boldsymbol{C}$ is a nonconstant polynomial, $V_{\alpha}=$ $\left\{z \in C^{n}: f(z)=\alpha\right\}$, and $\Phi$ is a biholomorphic map from $\boldsymbol{C}^{n}$ onto $\boldsymbol{C}^{n}$. If $\alpha_{1} \neq \alpha_{2}$ and $\Phi\left(V_{\alpha_{i}}\right)=V_{\alpha_{i}}$ for $i=1,2$, then $\Phi\left(V_{\beta}\right)=V_{\beta}$ for every $\beta \in C$, and $f=f \circ \Phi$.

This theorem claims that if a biholomorphic map preserves two level sets of a polynomial, then it preserves all level sets. He shows that "polynomial" can not be replaced by "entire function". Compare this theorem with ours. Let $H: M \rightarrow \widetilde{M}$ be an algebraic CR diffeomorphism between algebraic CR manifolds whose slices and base spaces are all algebraic. Assume that $\left.H\right|_{N}: N \rightarrow \widetilde{N}$ is an algebraic CR diffeomorphism. Then we have the following question.

QUESTION 4.2. If there exist finite number of points $p_{1}, \ldots, p_{\lambda} \in N$ and $\tilde{p}_{1}, \ldots$, $\tilde{p}_{\lambda} \in \widetilde{N}$ such that $H\left(M\left(p_{j}\right)\right) \subset \widetilde{M}\left(\tilde{p}_{j}\right)$ for $j=1, \ldots, \lambda$, then, for any $p \in N$, does there exist $\tilde{p} \in \tilde{N}$ such that $H(M(p))=\tilde{M}(\tilde{p})$ holds ? If the statement is true for some $\lambda$, find the minimal number of $\lambda$. 
4.2. The Baracco-Zampieri theorem. Baracco and Zampieri proved, in some sense, CR geometry version of Hartogs' theorem in [5]. They consider a real analytic foliation of $\boldsymbol{C}^{n}$ by complex analytic manifolds of dimension $m$ issued transversally from a generic manifold $M \subset C^{n}$ of codimension $m$. Then they proved that any continuous function which is a CR function on $M$ and has separate holomorphic extension along each leaf is holomorphic. Let $M$ and $\tilde{M}$ be the same as in Theorem 1.2. We have the following question.

QUESTION 4.3. Assume that $F: N \rightarrow \widetilde{N}$ is a CR homeomorphism and that, for each $p \in N$, there exists $\tilde{p} \in \tilde{N}$ such that $F$ has a CR homeomorphic extension $F: M(p) \rightarrow$ $\widetilde{M}(\tilde{p})$. Then does $F$ have a CR homeomorphic extension $F: M \rightarrow \widetilde{M}$ ?

\section{REFERENCES}

[1] M. S. Baouendi, P. Ebenfelt And L. P. Rothschild, Real submanifolds in complex spaces and their mappings, Princeton Mathematical Series 47, Princeton University Press, Princeton, NJ, 1999.

[2] M. S. Baouendi, P. Ebenfelt And L. P. Rothschild, Rational dependence of smooth and analytic CR mappings on their jets, Math. Ann. 315 (1999), 205-249.

[ 3 ] M. S. Baouendi, P. Ebenfelt And L. P. Rothschild, Dynamics of the Segre varieties of a real submanifold in complex space, J. Algebraic Geom. 12 (2003), 81-106.

[ 4 ] T. Bloom And I. GRAHAm, On "type" conditions for generic real submanifolds of $\boldsymbol{C}^{n}$, Invent. Math. 40 (1977), 217-243.

[ 5 ] L. BARACCO AND G. ZAMPIERI, Separate holomorphic extension of CR function, Manuscripta Math. 128 (2009), 411-419.

[6] P. Ebenfelt And L. P. Rothschild, Transversality of CR mappings, Amer. J. Math. 128 (2006), no. 5, 1313-1343.

[ 7 ] K. FukUi, The necessary and sufficient condition for the group of leaf preserving diffeomorphisms to be simple, J. Math. Soc. Japan 64 (2012), no. 1, 181-184.

[ 8 ] A. Hayashimoto, One remark for CR equivalence problem, J. Korean Math. Soc. 37 (2000), no. 2, $245-251$.

[9] A. Hayashimoto, S. Y. Kim And D. Zaitsev, Decomposition of CR-Manifolds and Splitting of CR-maps, Ann. Sc. Norm. Super. Pisa Cl. Sci. (5) 2 (2003), no. 3, 433-448.

[10] W. Rudin, Preservation of level sets by automorphisms of $\boldsymbol{C}^{n}$, Indag. Mathem. (N.S.) 4 (1993), no. 4, 489497.

Nagano National College of Technology

716 TOKUMA, NAGANO 381-8550

JAPAN

E-mail address: atsushi@nagano-nct.ac.jp 